\title{
Cooperative Spatial Multiplexing for CR Users Sharing a Common Channel with Primary Users*
}

\author{
YANG XIAO, JINFENG KOU \\ Institute of Information Science \\ Beijing Jiaotong University \\ Beijing 100044,P.R. CHINA \\ Emails: yxiao@bjtu.edu.cn, xiaokouzi922@126.com
}

\begin{abstract}
To enable mobile stations (MSs) of secondary users (SUs) in cognitive radio (CR) networks sharing a common channel with MSs of primary users (PUs) is an important but challenging issue. In this paper, a cooperative spatial multiplexing (CSM) scheme is proposed, where base station (BS) in CR network has K antennas and each SU or PU has one antenna only, and BS supports PUs and SUs by K spatial channels. To ensure communication quality of the network, the paper applies LDPC as the channel coding for BS, PUs and SUs. Simulations verify the proposed scheme with good spatial multiplexing capacity and BER performance.
\end{abstract}

Keywords: wireless communication, cognitive MIMO, spatial multiplexing, encoding and decoding algorithms Received: May 5, 2021. Revised: November 28, 2021. Accepted: December 20, 2021. Published: December 31, 2021.

\section{Introduction}

The demand for high data rates in wireless networks significantly increased over the last few years and stimulated the interest in efficient utilization of so called multiple-input multiple-output systems (MIMO) [1-19]. By utilizing multiple antenna architectures at both BS and MSs of CR network, significantly higher channel capacities close to that of wired networks are possible [1, 2, 16-19]. While, there are two problems need to be solved: first one is co-channel interference for SUs' and PUs' in a network, since SUs' and PUs' channels are not sure to be orthogonal each other; second one is MSs implementation complexity [16-19]. In the uplink, sophisticated multiuser detection (MUD) can be implemented at the BSs to counter the co-channel interference, however in the downlink, the MS receivers are usually required to be simple. In particular, in broadcast scenarios (downlink situation), where no joint processing of the received signals is possible, MIMO precoding schemes in $[4,5]$ are attractive since they can be viewed as the counterpart to successive cancellation which is applicable in uplink scenarios only.

Different from classical MIMO, the existing cooperative spatial multiplexing (CSM) schemes for MIMO network [10-15] need several base stations (BSs) and mobile stations (MSs) to support one data transmitting. However, there are four vital problems for this scheme to hinder CSM's application. The first problem is the exact synchronization among the MSs and BSs; the second one is the spatial channels' estimation for BSs and MSs [20]. The third one is space-time coding and decoding for the BSs and MSs, every one of the MSs and BSs needs to know the spatial channels' parameters [16, 20]; and the fourth one is the complexity of the implementation systems and MIMO network. That is to

This work was supported by the Beijing Natural Science Foundation of China: (No. 4102050).

E-ISSN: 2224-3488 say, we can not follow the existing CSM schemes [10-15], and had to propose new CSM solutions. The proposed CSM systems including the MIMO base stations (BS) and MIMO MS are designed, while we can not apply the existing CSM schemes [10-15] simply because of the above problems.

The proposed cooperative spatial multiplexing systems in this paper are different from the existing CSM schemes [10-15]. First, in our CSM, one CR network has one BS and many MSs of (PUs and SUs), BS has K antennas, while each MS only has one antenna, MSs have not used MIMO technique: no space-time coding and decoding. Second, in the proposed scheme, the CSM provides the BS of $\mathrm{K}$ antennas to support K MSs by one frequency channel only, the existing CSM schemes have not considered our approach. Third, the proposed CSM scheme solves the co-channel interference in CR network by LDPC [21-25], but the existing CSM schemes have no ability to solve the problem. Fourth, there is the synchronization problem among the MSs and BSs of the existing CSM schemes, one BS can support $\mathrm{K}$ MSs with independent spatial channels. Fifth, contrary to the existing CSM, in the proposed CSM, MSs need not to estimate the spatial channels' parameters, which make their implementation simplified greatly.

The following contents are organized as following. The proposed CSM downlink and CSM uplink schemes based on MIMO-CR network are given in Section II and Section III, respectively. In Section IV, we give the simulation results of a MIMO-CR network. Finally, Section V concludes the paper.

\section{The Proposed Csm Downlink of Mimo-cr Network}

Consider the downlink channel of MIMO-CR network shown in Fig. 1. In the proposed MIMO CR systems, the BS 
in CR network uses $\mathrm{K}$ antennas to transmit and receive the MSs' data stream of real symbols. From Fig.1, we see that the $\mathrm{K}$ downlink paralleled sequences for $\mathrm{K}$ MSs are transmitted from K antennas of BS transmitter. Since BS has $\mathrm{K}$ antennas, BS can divide $\mathrm{K}$ MSs into SU group and PU group who share the same frequency bandwidth in CR network.

In Fig.1, to keep the MIMO channels be independent, the distance of two MSs is lager than 10 radio signal wavelengths, and the distance of two receiving antennas of BS is lager than 4 radio signal wavelengths, else the channel matrix may be singular.

The proposed CSM scheme in Fig. 1 can provide K spatial channels to support K MSs of PUs and SUs at one frequency band-width, while there is one PU only for existing CR network only at one frequency band-width, and all SUs had to back-off.

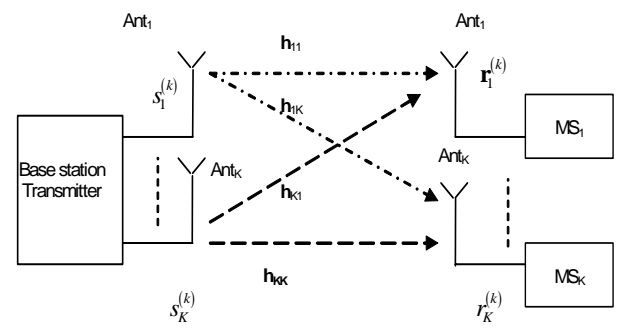

Fig. 1 The downlink channel of MIMO-CR network

We consider using MIMO precoding at the transmitter MIMO-CR BS, the purpose is to simplify the receiver implementation of MSs of PUs and SUs. The implementation complexity of proposed MIMO-CR systems is moved to BSs: downlink MIMO ZF precoding, so there is no space-time coding and decoding for MSs of PUs and SUs.

The proposed BS transmitter structure supports spatial multiplexing scheme for downlink transmission, and it is shown as in Fig. 2. In the proposed MIMO-CR network, MSs need not estimate the MIMO channel parameters, and they only need to provide BS an uplink pilot for $k$ th time-slot.

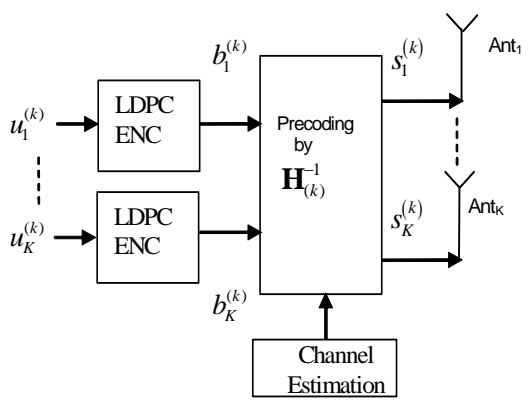

Fig. 2 MIMO precoding transmitter of BS for MSs
Fig. 2 only provides base-band processing blocks of the BS transmitter. Considering $\mathrm{K}$ MSs, the BS transmitter include $\mathrm{K}$ processing blocks as that in Fig.2. BS can determine the spatial multiplexing number by the number of PUs and MUs.

At downlink, firstly, BS's LDPC codec encodes the MSs data streams $\left\{u_{1}^{(k)}\right\}, \ldots,\left\{u_{K}^{(k)}\right\}$ of $k$ th time-slot into $K$ streams $\left\{b_{1}^{(k)}\right\}, \ldots,\left\{b_{K}^{(k)}\right\}$ for $\mathrm{MS}_{1}, \ldots, \mathrm{MS}_{\mathrm{K}}$.

Secondly, the LDPC streams $\left\{b_{1}^{(k)}\right\}, \ldots,\left\{b_{K}^{(k)}\right\}$ of MSs are precoded by inversed channel matrix as a linear combination of the K sub-streams $S_{1}^{(k)}, \ldots, S_{K}^{(k)}$,

$\left[\begin{array}{c}s_{1}^{(k)} \\ \vdots \\ s_{K}^{(k)}\end{array}\right]=\left[\begin{array}{ccc}h_{11}^{(k)} & \ldots & h_{1 K}^{(k)} \\ \vdots & \ddots & \vdots \\ h_{K 1}^{(k)} & \ldots & h_{K K}^{(k)}\end{array}\right]^{-1}\left[\begin{array}{c}b_{1}^{(k)} \\ \vdots \\ b_{K}^{(k)}\end{array}\right]$

where $h_{i j}^{(k)}, i, j \in\{1, \ldots, K\}$ denote the channel coefficient that the path from the transmitter antenna $i$ to the MS's antenna $j$ experiences independent Rayleigh fading, shown in Fig.1, and we let

$\mathbf{H}_{(k)}=\left[\begin{array}{ccc}h_{11}^{(k)} & \ldots & h_{1 K}^{(k)} \\ \vdots & \ddots & \vdots \\ h_{K 1}^{(k)} & \ldots & h_{K K}^{(k)}\end{array}\right]$

In the proposed BS system, the channel parameters of $\mathbf{H}_{(k)}^{-1}$ are provided by the channel estimation model of BS, shown in Fig.2.

BS in the proposed scheme accesses the MSs according to the spatial channels to be independent for the K MSs, which requires $\mathbf{H}_{(k)}$ should be full rank. This is always possible since the number of active MSs at different positions in CR network is always larger than K. The BS only does not select the MSs to be near each other [16].

In the proposed scheme, we need not require the MSs to estimate the channel parameters, since it will greatly increase the implementation complexity of MSs. The channel estimation is arranged on BS utilizing the approach of [9].

The received signals at the antennas of MSs are given by

$\left[\begin{array}{c}r_{1}^{(k)} \\ \vdots \\ r_{K}^{(k)}\end{array}\right]=\left[\begin{array}{ccc}h_{11}^{(k)} & \ldots & h_{1 K}^{(k)} \\ \vdots & \ddots & \vdots \\ h_{K 1}^{(k)} & \ldots & h_{K K}^{(k)}\end{array}\right]\left[\begin{array}{c}s_{1}^{(k)} \\ \vdots \\ s_{K}^{(k)}\end{array}\right]+\left[\begin{array}{c}n_{1}^{(k)} \\ \vdots \\ n_{K}^{(k)}\end{array}\right]$

where $h_{i j}^{(k)}, i, j \in\{1, \ldots, K\}$ denote the channel coefficient that the path from the transmitter antenna $i$ of BS to the receiver antenna of MS $j$ experiences independent Rayleigh fading. The receiver noises $n_{j}^{(k)}, j \in\{1, \ldots, K\}$ of the 
antenna $j$ are normalized to be $C N(0,1)$ (zero-mean unit-variance complex Gaussian).

Since the precoding at BS is adopted, the received signals $r_{i}^{(k)}, i=1, \ldots, K$, from the MSs' antennas, are independent, and can be further written as

$$
\begin{aligned}
& {\left[\begin{array}{c}
r_{1}^{(k)} \\
\vdots \\
r_{K}^{(k)}
\end{array}\right]=\left[\begin{array}{ccc}
h_{11}^{(k)} & \ldots & h_{1 K}^{(k)} \\
\vdots & \ddots & \vdots \\
h_{K 1}^{(k)} & \ldots & h_{K K}^{(k)}
\end{array}\right]\left[\begin{array}{ccc}
h_{11}^{(k)} & \ldots & h_{1 K}^{(k)} \\
\vdots & \ddots & \vdots \\
h_{K 1}^{(k)} & \ldots & h_{K K}^{(k)}
\end{array}\right]^{-1}\left[\begin{array}{c}
b_{1}^{(k)} \\
\vdots \\
b_{K}^{(k)}
\end{array}\right]+\left[\begin{array}{c}
n_{1}^{(k)} \\
\vdots \\
n_{K}^{(k)}
\end{array}\right]} \\
& =\left[\begin{array}{c}
b_{1}^{(k)} \\
\vdots \\
b_{K}^{(k)}
\end{array}\right]+\left[\begin{array}{c}
n_{1}^{(k)} \\
\vdots \\
n_{K}^{(k)}
\end{array}\right]
\end{aligned}
$$

From (4), the proposed MS receiver can be very simple, whose base-band circuit is shown in Fig.3. The soft information $r_{i}^{(k)}$ needs to be decoded by LDPC decoder in

Fig.3 [21-25].

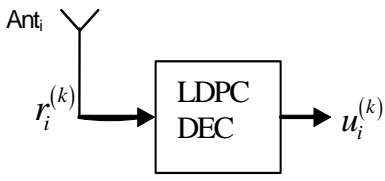

Fig. 3 The MS receivers of MIMO CR network

The proposed CSM downlink scheme of MIMO CR system, the MSs need not estimate the spatial channel parameters, the base station of $\mathrm{CR}$ network provides $\mathrm{K}$ spatial channels for K MSs. This is one of main contributions of the proposed CSM scheme. However, in the downlink of wireless network, the existing CSM receivers need to joint-recover the transmitted data streams from BS by channel matrices, and MSs had to estimate Rayleigh channel parameters by the pilot signals of BS [10].

\section{The Proposed Csm Uplink of Mimo-cr Network}

Considering the uplink of the CSM MIMO-CR network shown as in Fig.4, where there are $\mathrm{K}$ antennas at BS but there is one antenna of at each MS, assuming there are $\mathrm{K}$ active MSs in the network. The MSs transmit the K streams through its single transmitting antenna through the flat fading channels in the MIMO-CR network. The proposed BS assigns spatial channels to the MSs of PUs and SUs according to their spatial positions, and MSs should not near each other.

The proposed MS transmitter of MIMO-CR network is shown in Fig.5. At the uplink, firstly, MS's LDPC codec encodes its data streams $\left\{u_{i}^{(k)}\right\}, i=1, \ldots, K$ into LDPC streams $\left\{b_{i}^{(k)}\right\}, i=1, \ldots, K$ [21-25]. Then, the LDPC streams are transmitted different MSs, and they are received through Rayleigh fading channel by BS.

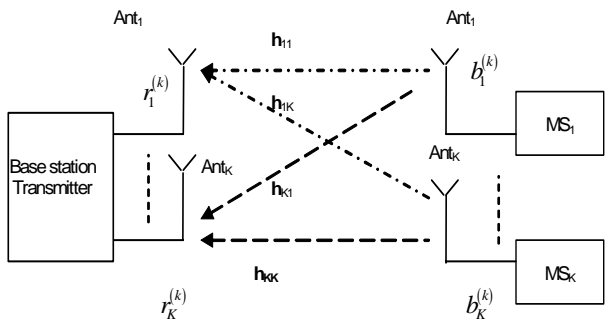

Fig. 4 the uplink of the CSM MIMO network

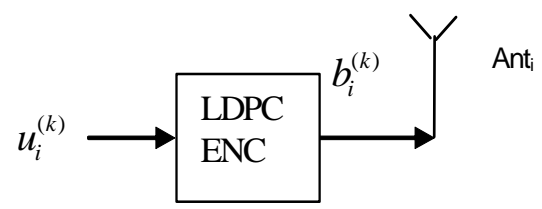

Fig.5 The MS transmitter of MIMO-CDMA network

Different from the existing CSM schemes for MSs, from Fig.5, we see that the proposed MS transmitter has no space-time coding. The CSM gain is obtained by the proposed BS MIMO receiver.

The proposed MIMO-CR BS receiver K MSs is shown in Fig. 6. In the proposed CSM scheme, each MS provides an uplink pilot sequence to BS. BS obtains the spatial channel parameters by the MS's pilot [9], this is different from the other existing CSM scheme.

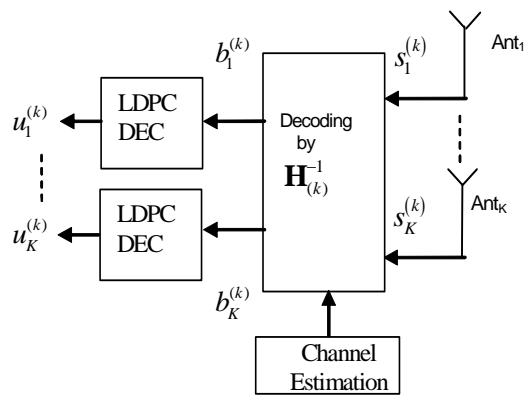

Fig. 6 MIMO receiver of MIMO-CD BS

The received signals at $\mathrm{K}$ antennas of BS are given by

$$
\left[\begin{array}{c}
r_{1}^{(k)} \\
\vdots \\
r_{K}^{(k)}
\end{array}\right]=\left[\begin{array}{ccc}
h_{11}^{(k)} & \ldots & h_{1 K}^{(k)} \\
\vdots & \ddots & \vdots \\
h_{K 1}^{(k)} & \ldots & h_{K K}^{(k)}
\end{array}\right]\left[\begin{array}{c}
b_{1}^{(k)} \\
\vdots \\
b_{K}^{(k)}
\end{array}\right]+\left[\begin{array}{c}
n_{1}^{(k)} \\
\vdots \\
n_{K}^{(k)}
\end{array}\right]
$$

where $h_{i j}^{(k)}, i, j \in\{1, \ldots, K\}$ denote the channel coefficient that the path from the MS $i$ to the receiver antenna $j$ of BS experiences independent Rayleigh fading. The receiver noise 
vectors $n_{j}^{(k)}, j=1, \ldots, K$ of the antenna $j$ are normalized to be $C N(0,1)$ (zero-mean unit-variance complex Gaussian).

From $r_{i}^{(k)}, i=1, \ldots, K$, the MIMO decoding model recovers $\left\{z_{i}^{(k)}\right\}, i=1, \ldots, K$, the LDPC coded streams with channel noise by using $\mathbf{H}_{(k)}^{-1}$,

$\left[\begin{array}{c}z_{1}^{(k)} \\ \vdots \\ z_{K}^{(k)}\end{array}\right]=\left[\begin{array}{ccc}h_{11}^{(k)} & \ldots & h_{1 K}^{(k)} \\ \vdots & \ddots & \vdots \\ h_{K 1}^{(k)} & \ldots & h_{K K}^{(k)}\end{array}\right]^{-1}\left(\left[\begin{array}{ccc}h_{11}^{(k)} & \ldots & h_{1 K}^{(k)} \\ \vdots & \ddots & \vdots \\ h_{K 1}^{(k)} & \ldots & h_{K K}^{(k)}\end{array}\right]\left[\begin{array}{c}b_{1}^{(k)} \\ \vdots \\ b_{K}^{(k)}\end{array}\right]+\left[\begin{array}{c}n_{1}^{(k)} \\ \vdots \\ n_{K}^{(k)}\end{array}\right]\right)$

$=\left[\begin{array}{c}b_{1}^{(k)} \\ \vdots \\ b_{K}^{(k)}\end{array}\right]+\left[\begin{array}{ccc}h_{11}^{(k)} & \ldots & h_{1 K}^{(k)} \\ \vdots & \ddots & \vdots \\ h_{K 1}^{(k)} & \ldots & h_{K K}^{(k)}\end{array}\right]^{-1}\left[\begin{array}{c}n_{1}^{(k)} \\ \vdots \\ n_{K}^{(k)}\end{array}\right]$

where $z_{i}^{(k)}, i=1, \ldots, K$ need to be decoded into $u_{i}^{(k)}, i=1, \ldots, K$ by LDPC decoder of the BS [21-25], which is shown in Fig.6.

\section{Simulations}

We assume that in a MIMO-CR network, there are one BS and four MSs (one is PU, three are SUs), BS has 4 antennas, and each MS has one antenna only, BS obtains the perfect channel estimation by the approach of [9], and the transmitted signals are modulated by BPSK.

To verify the LDPC role [21-25] in the proposed CSM system of MIMO-CR network to be valid, we simulate the downlink and uplink of MIMO-CR network with LDPC in MIMO-CR network. In following two experiments, we use the same QC LDPC codes in [21], in our system simulation, $p=127, a=2$ and $b=7$. Thus, the QC code has the code rate $1 / 2$, and code length to be 1270 .

Experiment 1: BER simulation of the proposed downlink MIMO-CR system with LDPC. BS had to use the proposed CSM to let one frequency band-width to support one PU and three SUs.

In the downlink network's simulation, the two systems (one with LDPC, the other no) transmit 200 data frames under same SNR and channel conditions, where $\mathbf{H}=\left[\begin{array}{cccc}0.9 & 0.31 & 0.29 & 0.13 \\ 0.19 & 0.78 & 0.4 & 0.21 \\ 0.24 & 0.3 & 0.71 & 0.33 \\ 0.11 & 0.17 & 0.4 & 0.83\end{array}\right]$.

The obtained BER results are shown in Fig. 7. Observing simulation results in Fig.7, we find that the PU and SUs have similar BER performance at MIMO-CR downlink, if BS has perfect channel estimation. When SNR is larger than 6 , the BER of PU and SUs is smaller than $10^{-5}$, which is expected by us. From (4), we also know that the precoding algorithm can complete the spatial separation for K MSs’ data screams. For the MSs, the received data is independent each other, and the multiuser interference has been removed due to (4).

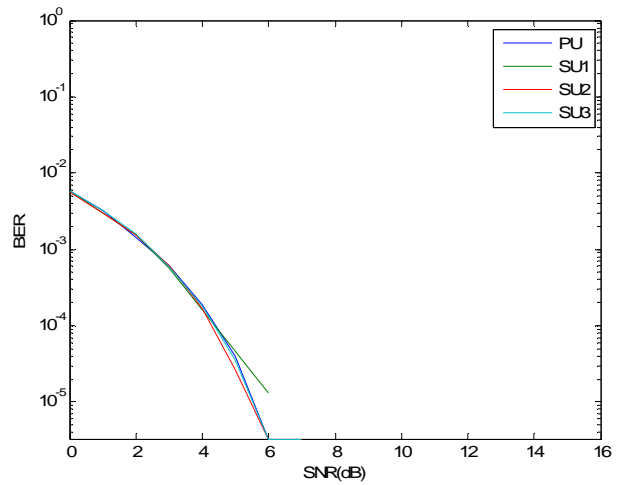

Fig. 7 The bit error rates (BER) of MIMO-CR downlink

Experiment 2: BER simulation of the proposed uplink MIMO-CR system with LDPC, the simulation parameters are same with Experiment 1 . The obtained BER results for PU and SUs are shown in Fig. 8.

Observing simulation results in Fig.8, we find that the PU MS has best BER performance, which in fact is determined by the channel matrix $\mathbf{H}$. When SNR is larger than $9 \mathrm{~dB}$, the BER of PU is smaller than $10^{-5}$, while BER of SU2 is smaller than $10^{-5}$, SNR needs to be larger than $15 \mathrm{~dB}$. The problem comes the second term of (6), where $\mathbf{H}^{-1}$ may enlarge the noise term $\left[\begin{array}{c}n_{1}^{(k)} \\ \vdots \\ n_{K}^{(k)}\end{array}\right]$ in (6), which is different from (4).

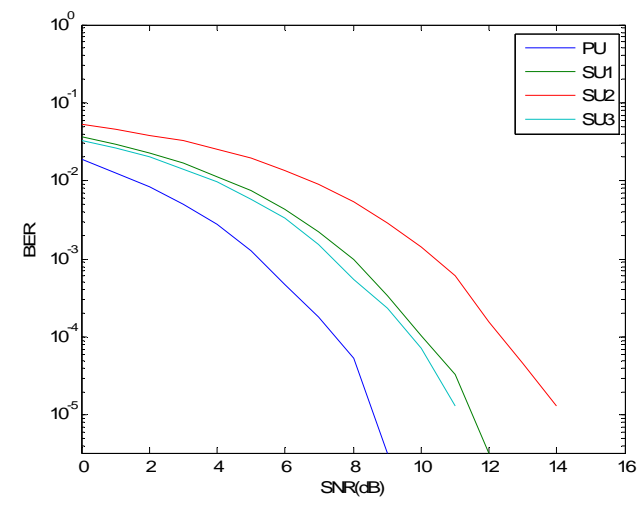

Fig.8 The bit error rates (BER) of MIMO CR uplink

\section{Conclusions}

In this paper, we proposed a CSM system with inversed channel matrices precoding and decoding algorithms for MIMO-CR network to enable MSs of PUs and SUs sharing a common channel. The CSM framework and algorithms of downlink and uplink are provided. It is possible to make PUs and SUs work at independent spatial channels in the 
MIMO-CR downlink and uplink. The simulation results showed that the proposed MIMO-CR network can achieve the good error rate performance in Rayleigh channels. Because MS has no complicated matrix decoding and channel estimation, the implementation of MSs becomes easy and can be accepted in future MIMO-CR network.

\section{References}

[1] I. E. Telatar, "Capacity of multi-antenna Gaussian channels," Euro.Trans. Telecom., vol. 10, pp. 585-595, Nov. 1999.

[2] G. J. Foschini and M. J. Gans, "On limits of wireless communications in a fading environment when using multiple antennas,” Wireless Personal Commun., vol. 6, pp. 311-335, 1998.

[3] G. G. Raleigh and J. M. Cioffi, "Spatio-temporal coding for wireless communications,” IEEE Trans. Commun., vol. 46, pp. 357-366, Mar. 1998.

[4] R. F. H Fischer, C. A. Windpassinger, "Improved MIMO precoding for decentralized receivers resembling concepts from lattice reduction”, 2003 IEEE Global Telecommunications Conference, (GLOBECOM '03), 1-5 Dec. 2003, Volume 4:1852 -1856.

[5] Y. Xiao, Y. Zhao and M. H. Lee, "MIMO Precoding of CDMA Systems”, Proc. 8th International Conference on Signal Processing, Beijing, 2006, Vol. 1: pp.397-401.

[6] Y. Xiao and M. H. Lee, "MIMO Multiuser Detection for CDMA Systems”, Proc. 8th International Conference on Signal Processing, Beijing, 2006, Vol. 1:pp.381-384.

[7] Y. Hou, Y. Xiao, "Cancelling Co-Channel Interference for MIMO-OFDM Systems Based on DCT and LDPC”, 2008 IET International Conference on Wireless Mobile \& Multimedia Networks, 2008, pp.268-271.

[8] Y. Xiao and M -H Lee, "Low Complexity MIMO-LDPC CDMA Systems over Multipath Channels", IEICE TRANS. COMMUN., VOL. E89-B, No. 5 May 2006, pp.1713-1717.

[9] Y. Xiao, J. L. Liu, H. J. Yin, K. Kim, MIMO Spatial Multiplexing Systems with Uplink Pilot and LDPC Codec, 5th International Conference on Wireless Communications, Networking and Mobile Computing, 2009. WiCom '09. 24-26 Sept. 2009, pp.1-5.

[10] F. Verde, D. Darsena and A. Scaglione, "Cooperative Randomized MIMO-OFDM Downlink for Multicell Networks: Design and Analysis”, IEEE TRANSACTIONS ON SIGNAL PROCESSING, VOL. 58, NO 1, pp.384-402, 2010.

[11] H. Skjevling, D. Gesbert, "Precoded Distributed Space-Time Block Codes in Cooperative Diversity-Based Downlink", IEEE Transactions on Wireless Communications, Vol. 6, No. 12, pp. 4209-4212, 2007.

[12] A. Özgür, O. Lévêque and D. N. C. Tse, "Hierarchical Cooperation Achieves Optimal Capacity Scaling in Ad Hoc Networks", IEEE Transactions on Information Theory, Vol. 53, No. 10, pp.3549-3572, 2007.

[13] O. Simeone, O. Somekh, H. V. Poor, S. Shamai, Distributed MIMO in Multi-Cell Wireless Systems via Finite-Capacity Links, ISCCSM 2008, Malta, 12-14 March 2008, pp.203-206.

[14] Hilde Skjevling, David Gesbert, Are Hjorungnes, "Receiver-Enhanced Cooperative Spatial Multiplexing with Hybrid Channel Knowledge”, Proc. IEEE Conference on Acoustics, Speech, and Signal Processing, ICASSP 2006, (Toulouse, France), IEEE, 14-19 May 2006. IV:65-68

[15] S. H. Lee and S. Y. Chung, "Degrees of Freedom of Cooperative MIMO in Cellular Networks", Proceedings of IEEE International Conference on Communications (IEEE ICC 2009), 2009, pp.1-5.

[16] Y. Xiao, MIMO Multi-antennas Wireless Communication Systems, Press of Posts and Telecommunications, Beijing, 2009.

[17] Hochwald, T. L. Marzetta, C. B. Papadias, A Transmitter Diversity Scheme for Wideband CDMA Systems Based on Space-Time Spreading, IEEE Journal on Selected Areas In Communications, 2001, 19(1): 48-60.

[18] Y. Xiao, K. S. Kim, G. Z. Qu. A Cognitive Spatial Multiplexing Scheme for MIMO-CDMA Networks. Proc. of Conference on
Wireless, Mobile and Multimedia Networks, Beijing, China, 2010: 147-150.

[19] Y. Xiao, Y. K. Zhang and G. Z. Qu, et al. Spatial Multiplexing Algorithms of Cognitive Base-Stations. Proc. of Conference on Wireless, Mobile and Multimedia Networks, Beijing, China, 2010: 221-224.

[20] W. -J. Chen, Y. Xiao and Y. Zhao, The algorithm implement of WCDMA channel estimation, 7th International Conference on Signal Processing Proceedings, 2004, v3: 1894-1897.

[21] Y. Xiao and M. H. Lee, Construction of good quasi-cyclic LDPC codes, Proceedings of IET International Conference on Wireless Mobile and Multimedia Networks (ICWMMN '06), Hangzhou, China, Nov. 2006, pp.172-176.

[22] Y. Xiao, K. Kim, Alternative good LDPC codes for DVB-S2, Proc. 9th International Conference on Signal Processing (ICSP 2008), Beijing, Oct. 26-29, 2008, pp.1959-1962.

[23] Y. Xiao, K. Kim, Good encodable irregular quasi-cyclic LDPC codes, 11th IEEE Singapore International Conference on Communication Systems (ICCS 2008), 19-21 Nov. 2008, pp.1291-1296.

[24] Y. Xiao, Evaluations of good LDPC codes based on generator matrices, Proc. of 2006 8th International Conference on Signal Processing, Beijing, China, Nov.16-20, 2006. pp. 2187-2190.

[25] Y. Xiao, Turbo and LDPC Codecs and their Applications, Press of Posts and Telecommunications, Beijing, 2010.

\section{Creative Commons Attribution License 4.0 (Attribution 4.0 International, CC BY 4.0)}

This article is published under the terms of the Creative Commons Attribution License 4.0 https://creativecommons.org/licenses/by/4.0/deed.en US 